\title{
Predictors of Malignancy in "Pure" Branch-Duct Intraductal Papillary Mucinous Neoplasm of the Pancreas without Enhancing Mural Nodules on CT Imaging: A Nationwide Multicenter Study
}

Tae-Hyeon Kim ${ }^{1}$, Young-Sik Woo ${ }^{2}$, Hyung-Ku Chon ${ }^{1}$, Jin-Hyeok Hwang ${ }^{3}$, Kyo-Sang Yoo ${ }^{4}$, Woo-Jin Lee ${ }^{5}$, Kwang-Hyuck Lee ${ }^{6}$, Jong-Kyun Lee ${ }^{6}$, Seok-Ho Dong ${ }^{7}$, Chang-Hwan Park ${ }^{8}$, Eun-Taek Park ${ }^{9}$, Jong-Ho Moon ${ }^{10}$, Ho-Gak Kim ${ }^{11}$, Kwang-Bum Cho ${ }^{12}$, Hong-Ja Kim ${ }^{13}$, Seung-Ok Lee ${ }^{14}$, Young-Koog Cheon $^{15}$, Jeong-Mi Lee ${ }^{16}$, Jin-Woo Park ${ }^{17}$, and Myung-Hwan Kim ${ }^{18}$

${ }^{1}$ Department of Internal Medicine, Wonkwang University College of Medicine, Iksan, ${ }^{2}$ Department of Internal Medicine, Kangnam Sacred Heart Hospital, Hallym University College of Medicine, Seoul, ${ }^{3}$ Department of Internal Medicine, Seoul National University College of Medicine, Seongnam, ${ }^{4}$ Department of Internal Medicine, Hanyang University College of Medicine, Guri, ${ }^{5}$ Pancreatobiliary Cancer Clinic, Center for Liver Cancer, National Cancer Center, Goyang, ${ }^{6}$ Department of Internal Medicine, Sungkyunkwan University School of Medicine, ${ }^{7}$ Department of Internal Medicine, Kyung Hee University School of Medicine, Seoul, ${ }^{8}$ Department of Internal Medicine, Chonnam University College of Medicine, Gwangju, ${ }^{9}$ Department of Internal Medicine, Kosin University College of Medicine, Busan, ${ }^{10}$ Department of Internal Medicine, Soonchunhyang University School of Medicine, Bucheon, ${ }^{11}$ Department of Internal Medicine, Catholic University of Daegu School of Medicine, ${ }^{12}$ Department of Internal Medicine, Keimyung University School of Medicine, Daegu, ${ }^{13}$ Department of Internal Medicine, Dankook University College of Medicine, Cheonan, ${ }^{14}$ Department of Internal Medicine, Chonbuk National University Medical School, Jeonju, ${ }^{15}$ Digestive Disease Center, Department of Internal Medicine, Konkuk University Medical Center, Konkuk University School of Medicine, Seoul, ${ }^{16}$ Department of Public Health, Wonkwang University Graduate School, Iksan, ${ }^{17}$ Department of Internal Medicine, Hepatobiliary and Pancreas Center, International St. Mary's Hospital, Catholic Kwandong University College of Medicine, Incheon, and ${ }^{18}$ Department of Internal Medicine, Asan Medical Center, University of Ulsan College of Medicine, Seoul, Korea

See editorial on page 481.

Background/Aims: Presence of enhanced mural nodules, which can be visualized using computed tomography (CT), is one of high-risk stigmata in branch-duct intraductal papillary mucinous neoplasms (BD-IPMNs). Conversely, the absence of enhanced mural nodules on preoperative imaging does not exclude malignant risk. The present study aimed to investigate other morphological features as predictors of malignancy in "pure" BD-IPMNs without enhanced mural nodules on CT. Methods: This retrospective study included 180 patients with surgically confirmed "pure" BD-IPMNs of the pancreas and no enhanced mural nodules on preoperative CT. The study was conducted at 15 tertiary referral centers throughout South Korea. Univariate and multivariate analyses were used to identify significant predictors of malignancy. Results: BD-IPMNs with low-grade $(n=84)$ or moderate-grade $(n=76)$ dysplasia were classified as benign; those with highgrade dysplasia $(n=8)$ or invasive carcinoma $(n=12)$ were classified as malignant. The multivariate analysis revealed that cyst size $\geq 30 \mathrm{~mm}$ (odds ratio, 8.6; $p=0.001$ ) and main pancreatic duct diameter $\geq 5 \mathrm{~mm}$ (odds ratio, $4.1 ; p=0.01$ ) were independent risk factors for malignancy in "pure" BDIPMNs without enhanced mural nodules on CT. Endoscopic ultrasound detected enhanced mural nodules (6/82) that had been missed on CT, and two IPMNs with enhanced mural nodules were malignant. Conclusions: In patients with "pure" BD-IPMNs who have no enhanced mural nodules on CT, cyst size $\geq 30 \mathrm{~mm}$ and main pancreatic duct diameter $\geq 5 \mathrm{~mm}$ may be associated with malignancy. (Gut Liver 2018;12:583-590)

Key Words: Branch duct-IPMN; Neoplasms; Multicenter study; Mural nodule

\section{INTRODUCTION}

Intraductal papillary mucinous neoplasms (IPMNs) of the pancreas have variable malignant potential, ranging from premalignant intraductal lesions to malignant neoplasms associated with invasive carcinoma. Furthermore, the management

Correspondence to: Myung-Hwan Kim

Department of Internal Medicine, Asan Medical Center, University of Ulsan College of Medicine, 88 Olympic-ro 43-gil, Songpa-gu, Seoul 05505, Korea

Tel: +82-2-3010-3180, Fax: +82-2-479-0824, E-mail: mhkim@amc.seoul.kr

Received on December 28, 2017. Revised on January 21, 2018. Accepted on January 30, 2018. Published online June 22, 2018 pISSN 1976-2283 eISSN 2005-1212 https://doi.org/10.5009/gnl17582

(a) This is an Open Access article distributed under the terms of the Creative Commons Attribution Non-Commercial License (http://creativecommons.org/licenses/by-nc/4.0) which permits unrestricted non-commercial use, distribution, and reproduction in any medium, provided the original work is properly cited. 
of IPMNs of the pancreas is closely related to their malignant potential. IPMNs are classified into three types on the basis of which pancreatic duct are involved: main-duct (MD) IPMNs, branch-duct (BD) IPMNs, and mixed-type IPMNs. ${ }^{1,2}$ Most of clinicians, as well as the 2012 international consensus guideline (ICG), recommend surgical resection of MD-IPMNs that have a risk of malignancy $\geq 36 \%$. However, the management of BDIPMNs remains controversial because they have a low risk of malignancy and the different values of malignant potential reported in the literature.

In the 2006 ICG known as the Sendai consensus guideline for BD-IPMNs, the indications for surgery were (1) the presence of mural nodules, (2) cyst size $\geq 30 \mathrm{~mm}$, and (3) dilated main pancreatic duct (MPD). ${ }^{3}$ However, it transpired that these indications were too sensitive and were leading to unnecessary surgery. In the updated 2012 ICG for BD-IPMNs, a dilated MPD (5 to 9 $\mathrm{mm}$ in diameter) and a cyst size of $\geq 30 \mathrm{~mm}$ were reclassified as "worrisome features," and only the presence of enhanced mural nodules (EMNs) on computed tomography (CT), which constitute high-risk stigmata, remained the strongest indicator for resection without further testing. ${ }^{4}$ The 2012 recommendations have been supported by many studies indicating that the presence of EMNs is the most important predictor of malignancy. ${ }^{5-9}$ Despite this, Shimizu et al. ${ }^{5}$ reported that $9.4 \%$ of malignant IPMNs show no EMNs on CT. More recently, "flat type" BD-IPMNsthat is, BD-IPMNs without EMNs on radiological imaging or endoscopic ultrasound (EUS)-comprised 9.8\% (9/91) of invasive carcinomas in patients whose BD-IPMNs had been resected. Moreover, such BD-IPMNs had a higher recurrence rate and a worse 5-year survival rate than BD-IPMNs that had EMNs on CT. ${ }^{10}$ Therefore, the absence of EMNs on imaging studies does not exclude malignancy in BD-IPMNs, and additional factors predicting malignancy in BD-IPMNs without EMNs are required. However, so far, few studies have addressed predictive factors for malignancy in "pure" BD-IPMNs without EMNs. Thus, we designed the present multicenter study to elucidate malignant potential and risk factors for malignancy in "pure" BD-IPMNs without EMNs or solid masses on CT imaging.

\section{MATERIALS AND METHODS}

\section{Study population}

We identified 218 patients with BD-IPMNs that had undergone preoperative abdominal $\mathrm{CT}$ and pancreatic resection at 15 tertiary hospitals throughout South Korea between 2004 and 2012. The diagnosis of IPMN was confirmed in all cases using histological examination of the resected pancreatic specimen. Of these patients, we selected 185 who had pathologically confirmed BD-IPMNs without MPD involvement or EMNs on preoperative abdominal CT (Fig. 1). Of these 185 patients, five were excluded because they had no detailed pathologic results. Ultimately, data were available from 180 patients. Eighty-two patients of these 180 patient underwent EUS. Contrast-enhanced EUS was not applied in most of the cases. This database was then retrospectively analyzed and supplemented with a review of the patients' electronic medical records. The study protocol was approved by The Institutional Review Boards of the 15 hospitals. Importantly, the indications for surgery were inconsistent among the hospitals.

Routine preoperative examinations involved clinical evaluation, routine blood tests (including assessment of tumor markers), and contrast-enhanced abdominal CT. On CT images, any enhanced protrusion along the cystic wall that was visible on any phase of the dynamic study was defined as an EMN. MPD diameter and cyst size were recorded as the maximum dimensions measured on cross-sectional images of preoperative abdominal CT. The radiologists who confirmed this finding was blinded to patients' information and the histopathological findings. The following data were retrospectively analyzed: demographics, clinical and radiological information, operative management, and pathology. Age, sex, symptoms, biochemical laboratory data, tumor markers, and all available preoperative imaging results were analyzed to enable malignancy-predicting factors to be identified.

IPMNs were diagnosed in accordance with the 2010 World Health Organization criteria, ${ }^{11}$ which categorize IPMNs as having low-, moderate-, or high-grade dysplasia, or as being associated with invasive carcinoma. Different pathologists from each hospital that participated in this study performed this pathologic evaluation. For the purpose of our analysis, low- and intermediate-grade dysplasia was classified as benign, whereas high-grade dysplasia and invasive carcinoma were defined as malignant. This classification was based on the assumption that BD-IPMNs with high-grade dysplasia or invasive carcinoma should be surgically resected, as recommended by the ICG and by other investigators. ${ }^{3,4}$

\section{Statistical analyses}

Statistical analyses were performed using SPSS version 19.0 (IBM Corp., Armonk, NY, USA). Continuous variables are expressed as medians and ranges; they were compared using the Mann-Whiney U test. Categorical variables were compared using either the chi-square or Fisher exact probability tests. Multivariate logistic regression models were used to estimate the predictive value of each variable for BD-IPMN malignancy. Variables were included in the models if they (1) were known risk factors for malignancy in BD-IPMNs, or (2) showed EMNs that were readily discernible on either abdominal CT or EUS. The optimal cutoff points for discriminating between malignant and benign BD-IPMNs were sought for each predictive factor using receiver operating characteristic (ROC) curves that were generated by calculating the sensitivities and specificities at several predetermined cutoff points. The area under the ROC curve (AUC) expressed how well each given factor was able to dis- 
criminate between malignant and benign IPMNs. Higher values indicated better discrimination; that is, a value of 0.5 indicated no predictive discrimination, while a value of 1.0 indicated perfect separation of patients. ${ }^{12}$ The level of significance was set at $\mathrm{p}<0.05$. All $\mathrm{p}$-values were two sided.

\section{RESULTS}

\section{Patient characteristics and histopathological findings}

A total of 180 patients met the inclusion criteria and were included in this study. The baseline characteristics of the subjects are detailed in Table 1 . The male to female ratio was 0.7:1. The mean age at presentation was similar between men and women $(63.3 \pm 9.5$ years vs $64.5 \pm 10.2$ years, respectively). Overall, 114 patients (63.3\%) were asymptomatic, and there were no significant differences in the frequency of abdominal pain and or acute pancreatitis between benign and malignant tumors.

Among all patients, there were 84 (46.7\%) with low-grade dysplasia, 76 (42.2\%) with moderate-grade dysplasia, eight (4.4\%) with high-grade dysplasia, and 12 (6.7\%) with invasive carcinoma (Fig. 1). In 10 patients (5.6\%), ordinary pancreatic duct adenocarcinoma (PDAC) with concurrent BD-IPMNs was

Table 1. Clinical Characteristics of 180 Patients with BD-IPMNs

\begin{tabular}{|c|c|c|c|c|}
\hline Characteristic & Benign BD-IPMN $(n=160)$ & Malignant BD-IPMN $(n=20)$ & Total $(\mathrm{n}=180)$ & p-value \\
\hline Male sex & $64(40)$ & $7(35)$ & $71(39.4)$ & NS \\
\hline Age, median (range), yr & $63.3(34-87)$ & $63.5(44-83)$ & $63.3(30-87)$ & NS \\
\hline \multicolumn{5}{|l|}{ Clinical symptoms } \\
\hline Abdominal pain & $41(25.4)$ & $7(35)$ & 49 (27.2) & NS \\
\hline Jaundice & 0 & $1(5)$ & $1(0.5)$ & NS \\
\hline Acute pancreatitis & $14(8.75)$ & $2(10)$ & $16(8.9)$ & NS \\
\hline \multicolumn{5}{|l|}{ Location of cysts } \\
\hline Head & $83(51.9)$ & $11(55)$ & $94(52.2)$ & NS \\
\hline Body & $32(20.0)$ & $2(10)$ & $34(18.8)$ & NS \\
\hline Tail & $28(17.5)$ & $3(15)$ & $40(22.6)$ & NS \\
\hline Multifocal & $17(10.6)$ & $4(20)$ & $21(11.7)$ & NS \\
\hline Cyst size, mean (range), mm & $27(10-62)$ & $35.5(20-110)$ & $28(10-110)$ & 0.0002 \\
\hline Diameter of MPD, median (range), mm & $2.0(2-10)$ & $4.2(2-9)$ & $2(2-10)$ & 0.02 \\
\hline Concurrent PDAC distinct from IPMN & $6(3.8)$ & $4(20)$ & $10(5.6)$ & NS \\
\hline Serum CEA, mean (range), ng/mL & $2(0.2-114)$ & $2(1-23)$ & $2(0.2-114)$ & NS \\
\hline Serum CA 19-9, mean (range), U/mL & $8(1-473)$ & $9.5(1-473)$ & $8(1-473)$ & NS \\
\hline
\end{tabular}

Data are presented as number (\%).

BD-IPMNs, branch-duct-type intraductal papillary mucinous neoplasms; NS, not significant; MPD, main pancreatic duct; PDAC, pancreatic ductal adenocarcinoma; CEA, carcinoembryonic antigen; CA 19-9, carbohydrate antigen 19-9.

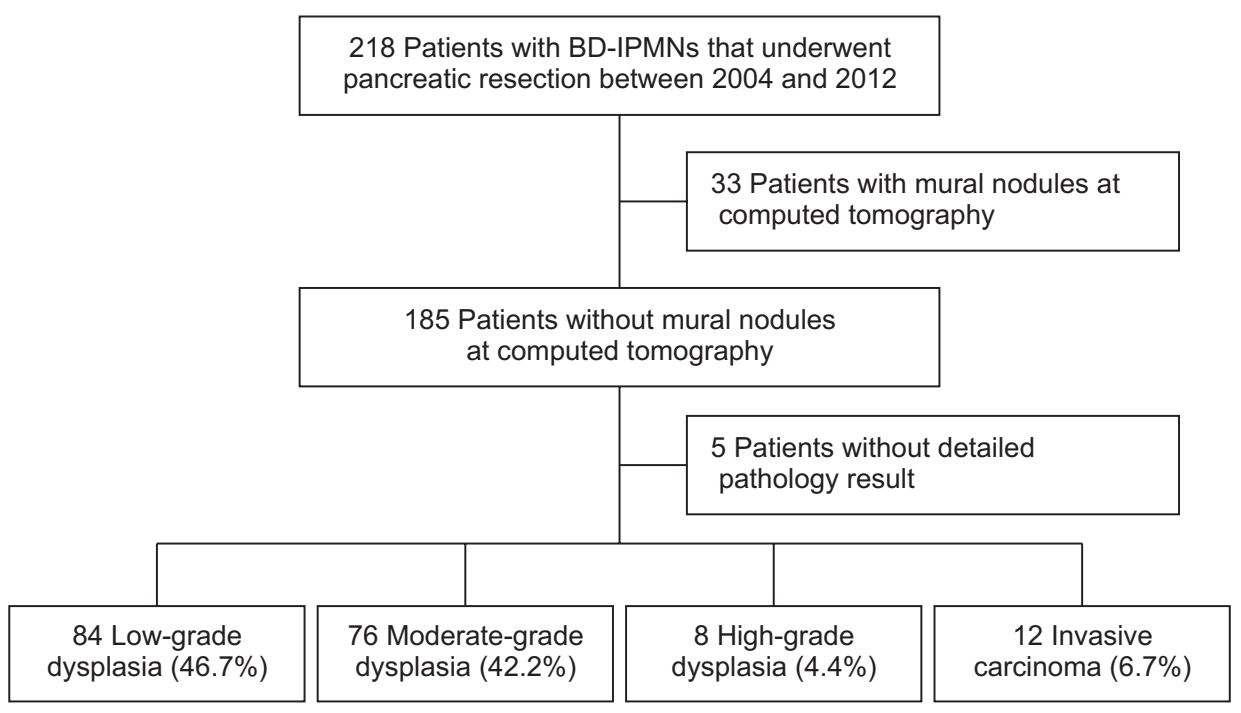

Fig. 1. Flow chart of study population recruitment and histologic distribution of resected branch-duct intraductal papillary mucinous neoplasms (BD-IPMNs) without mural nodules. 
found. The concurrent BD-IPMNs showed low-grade dysplasia in four patients, moderate-grade dysplasia in two patients, highgrade dysplasia in three patients, and invasive carcinoma in one patient.

The anatomic locations of the lesions, as determined using the preoperative images, are described in Table 1 . In 94 patients (52.2\%), the lesions were located at the head of the pancreas. The preoperative images showed that the mean cyst size was 28 $\mathrm{mm}$ (range, 10 to $110 \mathrm{~mm}$ ) and that the median diameter of the MPD was $2 \mathrm{~mm}$ (range, 2 to $10 \mathrm{~mm}$ ). The preoperative cyst size and MPD diameter differed significantly between benign and malignant neoplasms ( $\mathrm{p}=0.0002$ and $\mathrm{p}=0.02$, respectively). Pancreaticoduodenectomy, including both pylorus-preserving and pylorus-resecting forms, was performed in 81 patients (45.0\%); distal pancreatectomy was performed in 80 patients $(44.4 \%)$;

Table 2. Univariate Analysis of Factors Associated with Malignant BD-IPMNs

\begin{tabular}{lcccc}
\hline & $\begin{array}{c}\text { Benign } \\
\text { BD-IPMNs } \\
(\mathrm{n}=160)\end{array}$ & $\begin{array}{c}\text { Malignant } \\
\text { BD-IPMNs } \\
(\mathrm{n}=20)\end{array}$ & $\begin{array}{c}\text { Total } \\
(\mathrm{n}=180)\end{array}$ & $\mathrm{p}$-value \\
\hline Size of cyst $\geq 30 \mathrm{~mm}$ & $71(44.4)$ & $17(85)$ & $88(48.9)<0.0001$ \\
MPD diameter $\geq 5 \mathrm{~mm}$ & $25(15.6)$ & $7(35)$ & $25(13.9)$ & 0.03 \\
\hline
\end{tabular}

Data are presented as number (\%).

BD-IPMNs, branch-duct-type intraductal papillary mucinous neoplasm; MPD, main pancreatic duct.

Table 3. Multivariate Analysis of Factors Associated with Malignant BD-IPMNs

\begin{tabular}{lccc}
\hline & $95 \% \mathrm{CI}$ & Odds ratio & $\mathrm{p}$-value \\
\hline Size of cyst $\geq 30 \mathrm{~mm}$ & $2.3217-31.8518$ & 8.6 & 0.001 \\
MPD diameter $\geq 5 \mathrm{~mm}$ & $1.3342-12.3398$ & 4.1 & 0.01
\end{tabular}

BD-IPMNs, branch-duct-type intraductal papillary mucinous neoplasms; CI, confidence interval; MPD, main pancreatic duct. central pancreatectomy was performed in eight patients (4.4\%); enucleation was performed in seven patients (3.9\%), and total pancreatectomy was performed in four patients (2.2\%).

\section{Malignancy-predicting factors in all patients with BD- IPMNs: abdominal CT imaging}

As shown in Table 2, we found that the following two factors were significant predictors of malignancy on univariate analysis: cyst size $\geq 30 \mathrm{~mm}(\mathrm{p}<0.0001)$ and MPD dilatation $\geq 5$ $\mathrm{mm}(\mathrm{p}=0.03)$ on abdominal CT imaging. Multivariate logistic regression analyses were performed on these factors. The adjusted odds ratio (OR) for cyst size $\geq 30 \mathrm{~mm}$ on abdominal CT imaging was $8.6(p=0.001)$, while that for MPD diameter $\geq 5 \mathrm{~mm}$ was 4.1 ( $p=0.01$ ) (Table 3). To determine the cyst size cutoff point for differentiating between benign and malignant IPMNs, ROCs were conducted (Fig. 2). The AUC for the cyst size for malignant

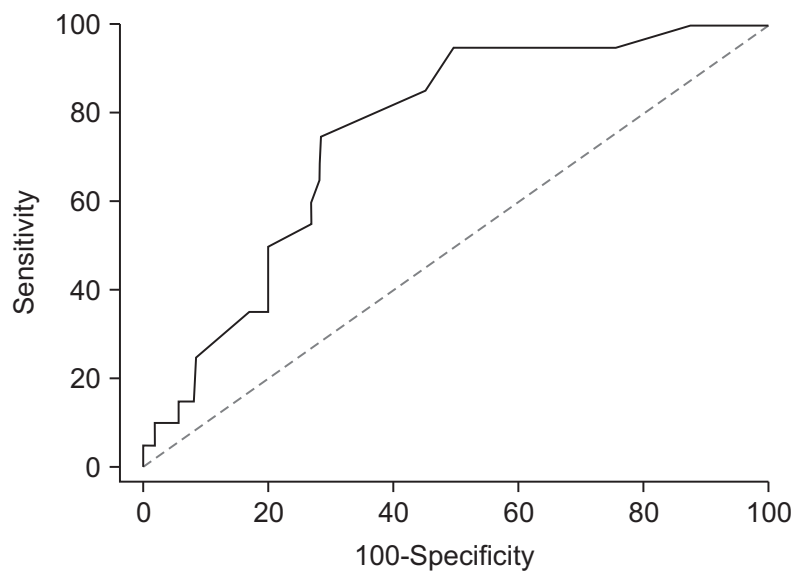

Fig. 2. Receiver operating characteristic curve for determining the cutoff point for cyst size on computed tomography. The area under the receiver operating characteristic curve for cyst size was $0.753(95 \%$ confidence interval, 0.677-0.834).

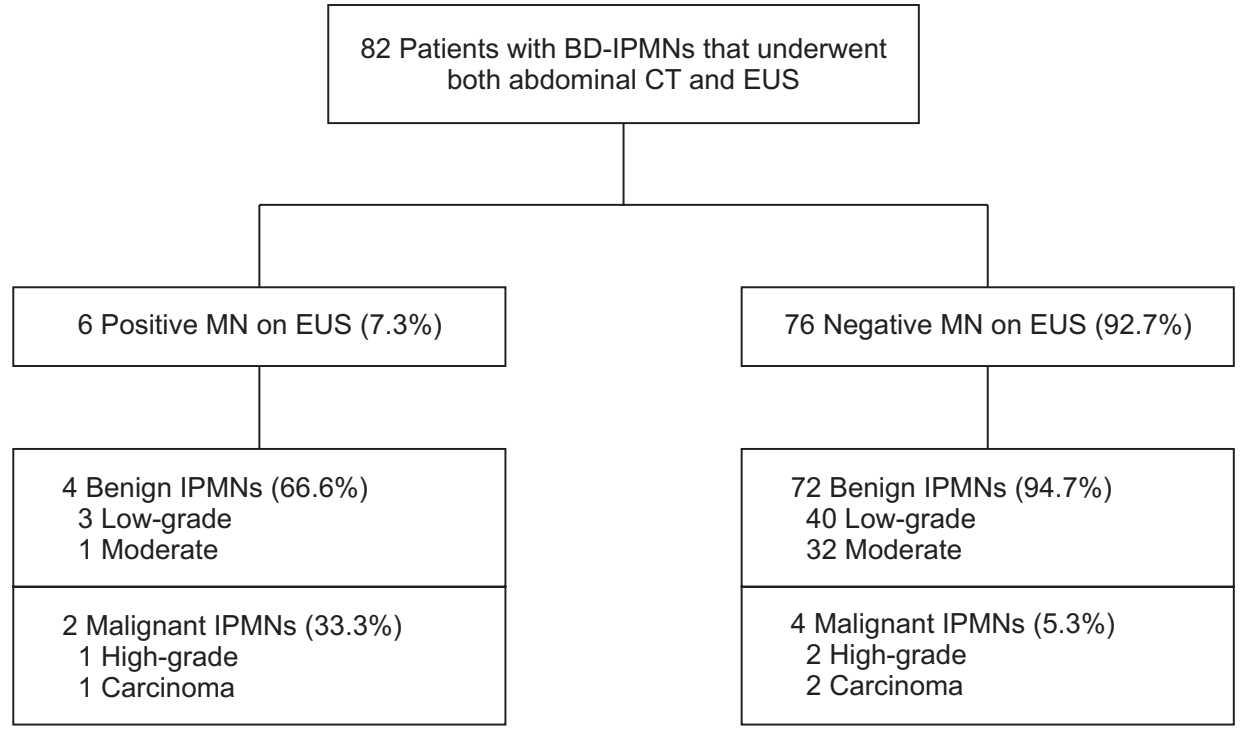

Fig. 3. Histological grade of branchduct intraductal papillary mucinous neoplasms (BD-IPMNs) in patients who underwent both abdominal computed tomography (CT) and endoscopic ultrasonography (EUS). MN, mural nodule. 
BD-IPMNs was 0.753 (95\% confidence interval [CI], 0.677 to 0.834 ); the sensitivity and specificity were $75 \%$ and $71 \%$, respectively, when the best cutoff point was set at a $30 \mathrm{~mm}$.

\section{Malignancy-predicting factors of patients with BD- IPMNs who underwent both EUS and CT}

In the subset of patients with BD-IPMNs ( $\mathrm{n}=82)$ who had undergone both EUS and abdominal CT, various clinical characteristics and EUS imaging factors were also analyzed. In six of these patients, EUS detected additional mural nodules (MNs) that had been missed on CT (7.3\%). Among these six patients, two (33.3\%) had malignant IPMNs. In patients who had cysts without MNs on EUS, the rate of malignant IPMNs was 5.3\% (high-grade dysplasia: two patients, invasive carcinoma: two patients) (Fig. 3). The grade of MN size in two patients with malignant BD-IPMN was $7 \mathrm{~mm}$ and $10 \mathrm{~mm}$, respectively. The only univariate predictor of malignant BD-IPMNs in this group was cyst size $\geq 3 \mathrm{~cm}(\mathrm{p}=0.01)$ and the presence of MN ( $p=0.01)$. Multivariate logistic regression analyses of those factors that were significant in univariate analysis revealed that the presence of MNs on EUS imaging were statistically significant predictors of BD-IPMN malignancy (OR, 9.0; $\mathrm{p}=0.02$ ) (Table 4).

\section{Diagnostic performance of independent malignant pre- dictors on diagnostic imaging}

We compared the diagnostic values of the factors in this study for differentiating between benign and malignant lesions (Table 5). Cyst size and "worrisome features" on CT imaging had high sensitivity (85\% and 95\%, respectively), low accuracy (58.9\% and 51.1\%, respectively), and low specificity (55.6\% and 46.3\%, respectively). The presence of MNs on EUS imaging

Table 4. Multivariate Analysis of Malignancy-Predicting Factors in Patients with BD-IPMNs Who Underwent both EUS and CT*

\begin{tabular}{cccc}
\hline & $95 \%$ CI & Odds ratio & p-value \\
\hline Presence of MN on EUS & $1.2506-64.7665$ & 9 & 0.02 \\
\hline
\end{tabular}

BD-IPMNs, branch-duct-type intraductal papillary mucinous neoplasms; EUS, endoscopic ultrasonography; CT, computed tomography; CI, confidence interval; MN, mural nodule.

*Preoperative EUS imaging was available for analysis in 82 patients. and MPD dilatation $\geq 5 \mathrm{~mm}$ on CT imaging had high specificity (94.7\% and 87.5\%, respectively) but low sensitivity (33.3\% and $20 \%$, respectively). MPD dilatation $\geq 5 \mathrm{~mm}$ and presence of MNs on EUS (80.6\% and 90.2\%, respectively) had higher accuracy than cyst size $\geq 30 \mathrm{~mm}$ and "worrisome features" (51.1\% and $58.9 \%$, respectively).

\section{DISCUSSION}

BD-IPMNs are less frequently associated with malignancy and carry a lower risk of progression toward malignancy than MD- or mixed type IPMNs. ${ }^{13}$ However, it remains challenging for clinicians to accurately identify BD-IPMNs with malignant pathology preoperatively. In the 2012 ICG, EMNs on CT implied a high-risk of malignancy in BD-IPMNs and were therefore an indication for surgery. However, in this regard, EMNs on CT showed high specificity and low sensitivity, meaning that physicians may miss a considerable number of malignant BDIPMNs. ${ }^{14-17}$ For this reason, clinicians must evaluate other morphological features, such as cyst size and MPD diameter, as predictors of malignancy in BD-IPMNs without EMNs on CT.

Cyst size is a key parameter that is emphasized in each version of the ICG. BD-IPMNs with cysts larger than $30 \mathrm{~mm}$ were considered candidates for surgical resection in the 2006 ICG. $^{3}$ In contrast, the 2012 ICG was more conservative, suggesting that BD-IPMNs larger than $30 \mathrm{~mm}$ without "high-risk stigmata" or "worrisome features" should be monitored closely, but not automatically resected. The guidelines also suggest that patients with a cyst $>30 \mathrm{~mm}$ in diameter, but without MNs on EUS, should undergo close surveillance rather than surgery. ${ }^{4}$ In the present study, in the case of BD-IPMNs, the sensitivity (85\%) and specificity (55.6\%) for differentiating between benign and malignant IPMNs was good when a cutoff value of $30 \mathrm{~mm}$ was used. Furthermore, we found that cyst size $\geq 30 \mathrm{~mm}$ was an independent risk factor for malignancy in BD-IPMNs without EMNs on CT. Similarly, Sahora et al. ${ }^{18}$ reported that patients with BD-IPMNs $\geq 30 \mathrm{~mm}$ in diameter had a higher incidence of malignancy than those with BD-IPMNs $<30 \mathrm{~mm}$ in diameter, even in the absence of other risk factors for malignancy. Several other recent studies have also reported that cyst size is a significant risk factor for

Table 5. Diagnostic Performance of Predictors of Malignancy in BD-IPMNs ( $\mathrm{n}=180)$

\begin{tabular}{lccccc}
\hline & Sensitivity (95\% CI) & Specificity (95\% CI) & Positive PV (95\% CI) & Negative PV (95\% CI) & Accuracy (\%) \\
\hline Worrisome features* $^{*}$ & $95(75.1-99.9)$ & $46.3(38.3-54.3)$ & $18.1(11.3-26.8)$ & $98.7(92.8-100)$ & 51.1 \\
Size of cyst $\geq 30 \mathrm{~mm}$ & $85(62.1-96.8)$ & $55.6(47.6-63.5)$ & $19.3(11.7-29.1)$ & $96.7(90.8-99.3)$ & 58.9 \\
MPD diameter $\geq 5 \mathrm{~mm}$ & $25(8.7-49.1)$ & $87.5(81.4-92.2)$ & $20(6.8-40.7)$ & $90.3(84.5-94.5)$ & 80.6 \\
Presence of MN on EUS & $33.3(4.3-77.7)$ & $94.7(87.1-98.5)$ & $33.3(4.3-77.7)$ & $94.7(87.1-98.5)$ & 90.2 \\
\hline
\end{tabular}

BD-IPMNs, branch-duct intraductal papillary mucinous neoplasms; CI, confidence interval; PV, predictive value; MPD, main pancreatic duct; MN, mural nodule; EUS, endoscopic ultrasonography.

*Worrisome features comprised pancreatic duct diameter $5-9 \mathrm{~mm}$ or cyst size $\geq 30 \mathrm{~mm}$; ${ }^{\dagger}$ Preoperative EUS imaging was available for analysis in 82 patients. 
malignancy in IPMNs. ${ }^{7,19,20}$ Relatedly, a recent systemic review and meta-analysis reported that low-risk and higher-risk IPMNs have an almost $8 \%$ and 25\% chance of progressing to pancreatic cancer within 10 years, respectively. ${ }^{21}$ According to the same study, surgical management should be considered, particularly in younger patients, when the cyst size is $>30 \mathrm{~mm}$, even when no other worrisome features are present. Nonetheless, a significant number of benign BD-IPMNs would be resected if surgery were performed on the basis of cyst size $\geq 30 \mathrm{~mm}$ alone.

Alternatively, MPD dilatation is a high-risk factors in predicting malignancy in IPMNs. ${ }^{22,23}$ Specifically, patients with a larger MPD diameter tend to have a higher incidence of malignant IPMNs (>3 to $4 \mathrm{~mm}$ : 36\%, >5 mm: 54\%-57\%, $\geq 10 \mathrm{~mm}$ : 60\%$63 \%) .{ }^{5,24-26}$ In a large cohort study of pathologically confirmed "pure" BD-IPMNs, including BD-IPMNs with EMNs, Ridtitid et al. ${ }^{27}$ showed that, among 135 resected BD-IPMNs, MPD dilation ( 5 to $9 \mathrm{~mm}$ ) was more frequently identified in malignant lesions than in benign lesions (50\% vs 18\%). However, with regards to "pure" BD-IPMNs without EMNs, few studies have addressed whether MPD diameter is related to malignancy. In one study by Koshita et al. ${ }^{10}$ involving patients with pathologically confirmed invasive cancer that was associated with BD-IPMNs without EMNs, $66 \%$ of the patients had an MPD of $\geq 5 \mathrm{~mm}$. However, the study had a relatively small sample size. ${ }^{10}$ Sadakari et al. ${ }^{20}$ evaluated 73 BD-IPMNs without EMNs, including six malignant IPMNs, and reported that an MPD diameter of $\geq 5 \mathrm{~mm}$ was significantly associated with malignancy. In the present study, an MPD of $\geq 5 \mathrm{~mm}$ was an independent malignant predictor in "pure" IPMNs without EMNs. Furthermore, the parameter had high specificity (87.5\%), even though the maximum MPD diameter of the enrolled patients was $10 \mathrm{~mm}$ (range, 2 to $10 \mathrm{~mm}$ ). Therefore, we tentatively suggest that MPD diameter $\geq 5 \mathrm{~mm}$ could be used as a predictor of malignancy in "pure" BD-IPMNs without EMNs.

In the revisions of 2012 ICG, the presence of "worrisome features" on CT indicated an evaluation using EUS to further verify the absence of MNs or measure the size of $\mathrm{MN}^{28}$ The worrisome features include cyst size $>3 \mathrm{~cm}$, enhancing $\mathrm{MN}<5$ $\mathrm{mm}$, enhancing cyst wall, main duct size of 5 to $9 \mathrm{~mm}$, and abrupt change in diameter of pancreatic duct. Zhong et al. ${ }^{16}$ reported that EUS in detecting true MNs have higher sensitivity (75\%) than CT (24\%). In the present study, MNs on EUS were an independent predictor of malignancy on multivariate analysis. Interestingly, we observed that EUS detected additional MNs $(6 / 82,7.3 \%)$ that had been missed on CT in patients who underwent both CT and EUS. Indeed, two such IPMNs with MNs on EUS imaging were malignant, suggesting that EUS affects decision-making in the case of many patients by detecting MNs that have been missed on CT imaging. In a previous study, the size of MNs $>5 \mathrm{~mm}$ on EUS, rather than just the presence of MNs, was an important predictor of malignancy in multivariate analysis, perhaps because smaller EMNs may represent mucin aggregates or dysplastic epithelium that has not yet reached advanced pathology, such as high-grade dysplasia or invasive carcinoma. ${ }^{17}$ Although no detailed size criterion has yet been defined as the most reliable predictor of malignancy in IPMNs, recent studies have suggested that increasing diameter of EMNs predict the specificity and accuracy of malignancy, with a cutoff value $10 \mathrm{~mm} \cdot{ }^{29,30}$ Currently, MNs can be distinguished from mucus aggregates within the IPMNs using contrast-enhanced harmonic EUS, which reportedly increases specificity for malignant nodules. ${ }^{31,32}$ Contrast-enhanced EUS was not applied in this study. Contrast enhanced EUS for evaluating MN in the pancreatic cyst is still under investigation and not recommended in the revisions of 2012 international consensus guideline and American Gastroenterology Association guideline. ${ }^{28,33}$

Although IPMNs are believed to be a direct precursor of PDAC, several retrospective series have documented a $4 \%$ to 9.2\% incidence of concurrent PDAC in a segment of the pancreas that is distant from the index IPMNs. ${ }^{1734-37}$ Additionally, several studies have shown that most concurrent PDACs are related to small BD-IPMNs without worrisome features. ${ }^{27,38}$ We observed concurrent PDACs that were distinct from the original BD-IPMNs in 10 patients (5.6\%). These results support the field carcinogenesis concept, which promotes development of IPMNs and PDAC. On the same note, Ideno et al. ${ }^{39}$ reported that patients with concomitant pancreatic cancer had a higher frequency of gastric subtype BD-IPMNs without guanine nucleotide binding protein (GNAS) mutation. Indeed, the epithelial subtypes of IPMNs may help to predict their tendency for malignant transformation (intestinal type) or development of concomitant pancreatic cancer (gastric type). The possibility of concurrent PDAC should always be considered carefully at the time of initial IPMN assessment. In this regard, EUS may be a crucial modality for detecting concurrent PDAC and EMNs that are invisible on CT scan. Relatedly, more effective strategies are needed during surveillance after resection (or observation without resection), because even 6-month interval surveillance may fail to diagnose PDAC at a sufficiently early stage.

There were several limitations associated with the current study. First, it was a retrospective evaluation of multicenter data. Therefore, since the data came from 15 centers, neither the imaging modalities nor the surgical indications were identical among the centers. This would likely have affected the results. Second, this study included only surgically resected cases, so many patients with small BD-IPMNs who had been conservatively followed up without surgery were not included. This patient selection may have affected the incidence of malignant BD-IPMNs. On the other hand, the malignant potential of BDIPMNs could be determined definitively in the present study without reference to the malignant potential of conservatively followed lesions, because a pathologic diagnosis was available for all BD-IPMNs. Third, Different pathologists from each hospital that participated in this study conducted pathologic 
assessment of IPMN according to the 2010 WHO classification. This problem may cause bias of dysplasia grade of IPMNs. Fourth, Preoperative EUS finding without contrast enhancement were evaluated for only 82 cases (45.6\%) of the 180 patients. This small number of patients who underwent EUS may lead to selection bias. The revisions of 2012 international consensus guideline recommend EUS for evaluating $\mathrm{MN}$ or main duct involvement of IPMN. ${ }^{28}$ Fifth, EUS-guided fine needle aspiration was not analyzed. In this regard, although the sensitivity and specificity of fluid analysis are not satisfactory in the case of IPMNs, the technique does provide further information by obtaining fluid that can be used for cytology, tumor marker assessment, amylase measurement, and DNA analysis. With regard to DNA analysis of aspirated fluid, new potential biomarkers that distinguish between benign and malignant IPMNs have been investigated and require further study. ${ }^{40}$

In conclusion, cyst size $\geq 30 \mathrm{~mm}$ and/or MPD diameter $\geq 5 \mathrm{~mm}$ in BD-IPMNs without EMNs on CT imaging may be optimal as worrisome features in the 2012 ICG guideline that require EUS examination and meticulous surveillance. Furthermore, more useful combinations of other predictive factors, such as novel biomarkers and cytological examination, should be developed in further studies to increase the accuracy of malignancy prediction for BD-IPMNs.

\section{CONFLICTS OF INTEREST}

No potential conflict of interest relevant to this article was reported.

\section{ACKNOWLEDGEMENTS}

This paper was supported by Wonkwang University in 2018.

\section{REFERENCES}

1. Furukawa T, Takahashi T, Kobari M, Matsuno S. The mucushypersecreting tumor of the pancreas: development and extension visualized by three-dimensional computerized mapping. Cancer 1992;70:1505-1513.

2. Kobari M, Egawa S, Shibuya K, et al. Intraductal papillary mucinous tumors of the pancreas comprise 2 clinical subtypes: differences in clinical characteristics and surgical management. Arch Surg 1999;134:1131-1136.

3. Tanaka M, Chari S, Adsay V, et al. International consensus guidelines for management of intraductal papillary mucinous neoplasms and mucinous cystic neoplasms of the pancreas. Pancreatology 2006;6:17-32.

4. Tanaka M, Fernández-del Castillo C, Adsay V, et al. International consensus guidelines 2012 for the management of IPMN and MCN of the pancreas. Pancreatology 2012;12:183-197.

5. Shimizu Y, Yamaue H, Maguchi H, et al. Predictors of malignancy in intraductal papillary mucinous neoplasm of the pancreas: analysis of 310 pancreatic resection patients at multiple high-volume centers. Pancreas 2013;42:883-888.

6. Seo N, Byun JH, Kim JH, et al. Validation of the 2012 international consensus guidelines using computed tomography and magnetic resonance imaging: branch duct and main duct intraductal papillary mucinous neoplasms of the pancreas. Ann Surg 2016;263:557-564.

7. Kim KW, Park SH, Pyo J, et al. Imaging features to distinguish malignant and benign branch-duct type intraductal papillary mucinous neoplasms of the pancreas: a meta-analysis. Ann Surg 2014;259:72-81.

8. Jang JY, Park T, Lee S, et al. Validation of international consensus guidelines for the resection of branch duct-type intraductal papillary mucinous neoplasms. Br J Surg 2014;101:686-692.

9. Hirono S, Tani M, Kawai M, et al. The carcinoembryonic antigen level in pancreatic juice and mural nodule size are predictors of malignancy for branch duct type intraductal papillary mucinous neoplasms of the pancreas. Ann Surg 2012;255:517-522.

10. Koshita S, Fujita N, Noda Y, et al. Invasive carcinoma derived from "flat type" branch duct intraductal papillary mucinous neoplasms of the pancreas: impact of classification according to the height of mural nodule on endoscopic ultrasonography. J Hepatobiliary Pancreat Sci 2015;22:301-309.

11. Bosman FT, World Health Organization; International Agency for Research on Cancer. WHO classification of tumours of the digestive system. 4th ed. Lyon: International Agency for Research on Cancer, 2010.

12. Harrell FE Jr, Califf RM, Pryor DB, Lee KL, Rosati RA. Evaluating the yield of medical tests. JAMA 1982;247:2543-2546.

13. Farrell JJ, Fernández-del Castillo C. Pancreatic cystic neoplasms: management and unanswered questions. Gastroenterology 2013;144:1303-1315.

14. Hirano S, Kondo S, Tanaka E, et al. Role of CT in detecting malignancy during follow-up of patients with branch-type IPMN of the pancreas. Hepatogastroenterology 2009;56:515-518.

15. Tanaka M. Controversies in the management of pancreatic IPMN. Nat Rev Gastroenterol Hepatol 2011;8:56-60.

16. Zhong N, Zhang L, Takahashi N, et al. Histologic and imaging features of mural nodules in mucinous pancreatic cysts. Clin Gastroenterol Hepatol 2012;10:192-198.

17. Kim TH, Song TJ, Hwang JH, et al. Predictors of malignancy in pure branch duct type intraductal papillary mucinous neoplasm of the pancreas: a nationwide multicenter study. Pancreatology 2015;15:405-410.

18. Sahora K, Mino-Kenudson M, Brugge W, et al. Branch duct intraductal papillary mucinous neoplasms: does cyst size change the tip of the scale? A critical analysis of the revised international consensus guidelines in a large single-institutional series. Ann Surg 2013;258:466-475.

19. Maguchi H, Tanno S, Mizuno N, et al. Natural history of branch duct intraductal papillary mucinous neoplasms of the pancreas: a 
multicenter study in Japan. Pancreas 2011;40:364-370.

20. Sadakari Y, Ienaga J, Kobayashi K, et al. Cyst size indicates malignant transformation in branch duct intraductal papillary mucinous neoplasm of the pancreas without mural nodules. Pancreas 2010;39:232-236.

21. Lee T, Kim HJ, Park SK, et al. Natural courses of branch duct intraductal papillary mucinous neoplasm. Langenbecks Arch Surg 2017;402:429-437.

22. Wakabayashi T, Kawaura Y, Morimoto H, et al. Clinical management of intraductal papillary mucinous tumors of the pancreas based on imaging findings. Pancreas 2001;22:370-377.

23. Yamaguchi K, Sugitani A, Chijiiwa K, Tanaka M. Intraductal papillary-mucinous tumor of the pancreas: assessing the grade of malignancy from natural history. Am Surg 2001;67:400-406.

24. Kim SC, Park KT, Lee YJ, et al. Intraductal papillary mucinous neoplasm of the pancreas: clinical characteristics and treatment outcomes of 118 consecutive patients from a single center. J Hepatobiliary Pancreat Surg 2008;15:183-188.

25. Schmidt CM, White PB, Waters JA, et al. Intraductal papillary mucinous neoplasms: predictors of malignant and invasive pathology. Ann Surg 2007;246:644-651.

26. Shimizu Y, Kanemitsu Y, Sano T, Senda Y, Mizuno N, Yamao K. A nomogram for predicting the probability of carcinoma in patients with intraductal papillary-mucinous neoplasm. World J Surg 2010;34:2932-2938.

27. Ridtitid W, DeWitt JM, Schmidt CM, et al. Management of branchduct intraductal papillary mucinous neoplasms: a large singlecenter study to assess predictors of malignancy and long-term outcomes. Gastrointest Endosc 2016;84:436-445.

28. Tanaka M, Fernández-Del Castillo C, Kamisawa T, et al. Revisions of international consensus Fukuoka guidelines for the management of IPMN of the pancreas. Pancreatology 2017;17:738-753.

29. Kobayashi G, Fujita N, Maguchi H, et al. Natural history of branch duct intraductal papillary mucinous neoplasm with mural nodules: a Japan Pancreas Society multicenter study. Pancreas 2014;43:532-538.

30. Kawada N, Uehara H, Nagata S, Tsuchishima M, Tsutsumi M, Tomita Y. Predictors of malignancy in branch duct intraductal papillary mucinous neoplasm of the pancreas. JOP 2014;15:459-464.

31. Ohno E, Itoh A, Kawashima H, et al. Malignant transformation of branch duct-type intraductal papillary mucinous neoplasms of the pancreas based on contrast-enhanced endoscopic ultrasonography morphological changes: focus on malignant transformation of intraductal papillary mucinous neoplasm itself. Pancreas 2012;41:855-862.

32. Kurihara N, Kawamoto H, Kobayashi Y, et al. Vascular patterns in nodules of intraductal papillary mucinous neoplasms depicted under contrast-enhanced ultrasonography are helpful for evaluating malignant potential. Eur J Radiol 2012;81:66-70.

33. Vege SS, Ziring B, Jain R, Moayyedi P; Clinical Guidelines Committee; American Gastroenterology Association. American gastroenterological association institute guideline on the diagnosis and management of asymptomatic neoplastic pancreatic cysts. Gastroenterology 2015;148:819-822.

34. Yamaguchi K, Ohuchida J, Ohtsuka T, Nakano K, Tanaka M. Intraductal papillary-mucinous tumor of the pancreas concomitant with ductal carcinoma of the pancreas. Pancreatology 2002;2:484490.

35. Uehara H, Nakaizumi A, Ishikawa 0, et al. Development of ductal carcinoma of the pancreas during follow-up of branch duct intraductal papillary mucinous neoplasm of the pancreas. Gut 2008;57:1561-1565.

36. Ingkakul T, Sadakari Y, Ienaga J, Satoh N, Takahata S, Tanaka M. Predictors of the presence of concomitant invasive ductal carcinoma in intraductal papillary mucinous neoplasm of the pancreas. Ann Surg 2010;251:70-75.

37. Tanno S, Nakano Y, Koizumi K, et al. Pancreatic ductal adenocarcinomas in long-term follow-up patients with branch duct intraductal papillary mucinous neoplasms. Pancreas 2010;39:36-40.

38. Tanno S, Nakano Y, Sugiyama Y, et al. Incidence of synchronous and metachronous pancreatic carcinoma in 168 patients with branch duct intraductal papillary mucinous neoplasm. Pancreatology 2010;10:173-178.

39. Ideno N, Ohtsuka T, Matsunaga T, et al. Clinical significance of GNAS mutation in intraductal papillary mucinous neoplasm of the pancreas with concomitant pancreatic ductal adenocarcinoma. Pancreas 2015;44:311-320.

40. Rockacy M, Khalid A. Update on pancreatic cyst fluid analysis. Ann Gastroenterol 2013;26:122-127. 